\title{
A Device for Indirect Measurement of Blood Pressure in Conscious Dogs
}

Short Report

\author{
Daijiro Horit and Takahiro Higuchi \\ Research Laboratory, Mitsubishi Yuka Pharmaceutical Co. \\ Ltd, Ami-cho, Ibaraki 300-03
}

\begin{abstract}
HoriI, D. and Higuchi, T. A Device for Indirect Measurement of Blood Pressure in Conscious Dogs. Tohoku J. exp. Med., 1985, 146 (4), 479-480 — A technique for indirect measurement of blood pressure by a combination of commercially available apparatuses was devised. There were good correlations in both of systolic and diastolic blood pressures measured by the indirect and direct methods.

- blood prressure; indirect method; dog
\end{abstract}

The tail-cuff method for measurement of blood pressure devised by Prioli and Winbury (1960) requires comparatively large scale shaving of fur in the tail. This is troublesome and it is desirable especially in a chronic toxicity test to minimize influences by the operation. We found that recording of pulstile deflections was obtainable more easily and more clearly than on the metacarpal pad covered with thin fur than on the portion of forelimb. As shown in Fig. 1, the indirect method devised utilizes a sphygmomanometer (Acoma) with an infant size pressure cuff, a photoelectric transducer (Nihon Kohden, MPP-3S) and a monitor such as an electrocardiograph or an oscilloscope. A pick up of a photoelectric transducer is placed on the depilated metacarpal pad and a pressure cuff is placed on the forelimb of dogs. As blood pressure in conscious animals is changeable, they must be placed in calm

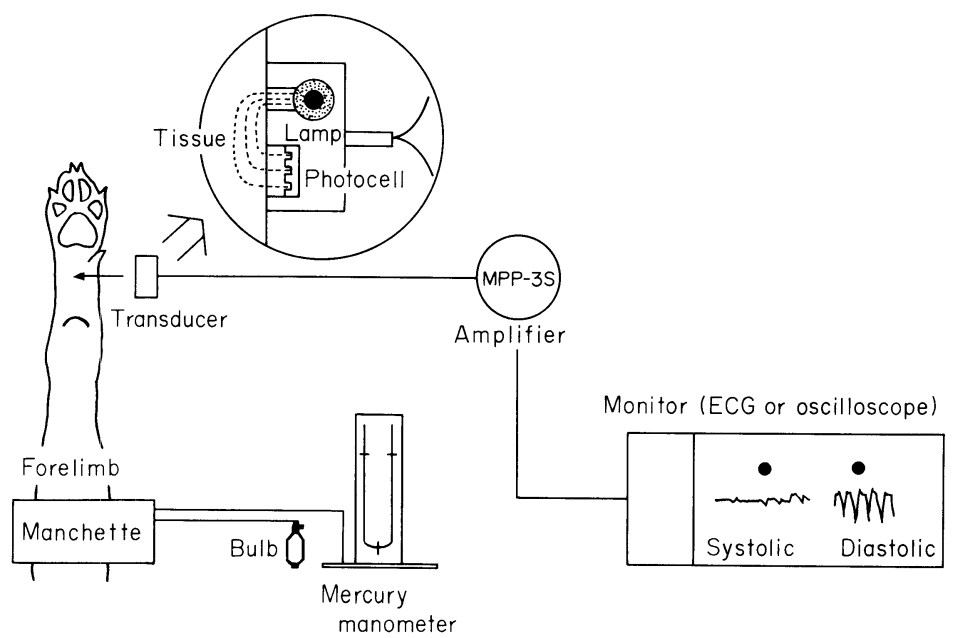

Fig. 1. Diagram of the device to measure blood pressure.

Received January 19, 1985; accepted for publication March 19, 1985 

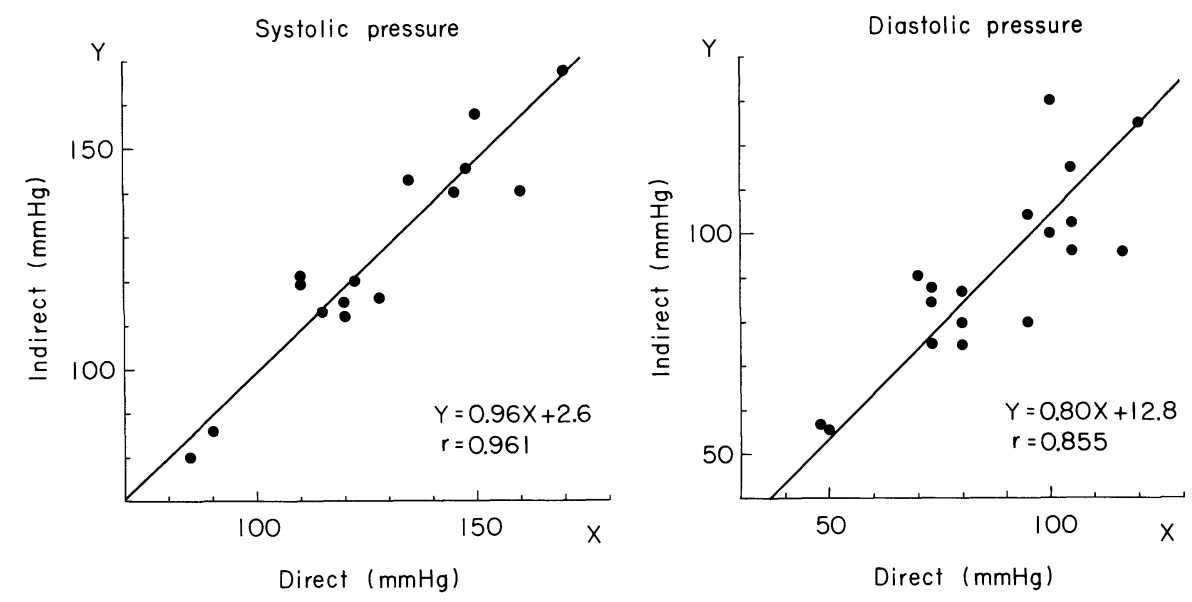

Fig. 2. Correlations in systolic and diastolic blood pressures measured by the indirect (vertical axis) and direct (horizontal axis) methods in 6 dogs.

conditions. To determine blood pressure, the pulse deflections must be clearly recorded. The pressure cuff is inflated until the deflections cease, then the pressure is reduced slowly (favorable reduction rate is about $1-2 \mathrm{mmHg} / \mathrm{sec}$ ). Systolic pressure is signaled by the appearance of pulse deflections. The point at which the amplitude of pulse deflections becomes to the value before the inflation of the pressure cuff represents diastolic pressure.

Femoral arterial pressure was also directly measured with percutaneous insertion of a needle (G-23) connected to an electronic manometer. The results of indirect measurement were compared with direct ones in 3 normotensive and 3 renal hypertensive conscious dogs (11-15 kg) over a wide range of pressure. Low blood pressure was produced by intravenous administration of hexamethonium. Fig. 2 shows that there were good correlations in blood pressure measured with the direct and indirect methods $(\mathrm{r}=0.96, p<0.01$ for systolic ; $\mathrm{r}=$ $0.86, p<0.01$ for diastolic). Systolic blood pressure is easily determined by an initial point of occurence of pulse wave, but diastolic one is somewhat difficult to determine. This method is favorable because it is inexpensive and easy to operate and gives no physical influences to the animals.

\section{Reference}

1) Prioli, N.A. \& Winbury, M.M. (1960) Indirect method for blood pressure determination in the dog. J. appl. Physiol. 15, 323-324. 